PAPER

\title{
Acute ophthalmoparesis in the anti-GQ1b antibody syndrome: electrophysiological evidence of neuromuscular transmission defect in the orbicularis oculi
}

\author{
Y L Lo, L L Chan, A Pan, P Ratnagopal
}

J Neurol Neurosurg Psychiatry 2004;75:436-440. doi: 10.1136/jnnp.2003.023630

See end of article for authors' affiliations

....................

Correspondence to: Dr Y L Lo, Department of Neurology, National

Neuroscience Institute, Singapore General Hospital, Outram Road, Singapore 169608; gnrlyl@sgh.com.sg

Received in revised form 16 July 2003

Accepted 16 July 2003

\begin{abstract}
Objective: To prospectively study anti-GQ1b antibody positive cases of acute ophthalmoparesis (AO) clinically and electrophysiologically.

Methods: Nine consecutive cases presenting with predominantly acute ophthalmoplegia were assessed clinically and had stimulated single fibre electromyography (SFEMG) of the orbicularis oculi at presentation. All had magnetic resonance imaging brain scans and anti-GQ1b antibody titres determined. Results: Four cases had elevated anti-GQ1b antibody titres and abnormal SFEMG studies, which improved in tandem with clinical recovery over three months. Five other anti-GQ1b antibody negative cases were diagnosed as diabetic related cranial neuropathy, idiopathic cranial neuropathy, ocular myasthenia gravis, and Tolosa-Hunt syndrome. All five cases showed complete recovery over a three month period.

Conclusions: This study demonstrated electrophysiologically the dynamic improvement of neuromuscular transmission of anti-GQ1b antibody positive cases of AO, in tandem with clinical recovery. SFEMG is of value in differentiating weakness due to neuromuscular transmission defect from neuropathy in these clinical situations.
\end{abstract}

$T$ he Miller Fisher syndrome (MFS) is an autoimmune condition characterised by ataxia, areflexia, and ophthalmoplegia. ${ }^{1}$ A large proportion of cases are associated with high titres of anti-GQlb antibodies. Acute ophthalmoparesis (AO) has been considered as a mild form of MFS or regional variant of the Guillain-Barré syndrome (GBS). ${ }^{2}$ There is increasing immunological evidence to suggest that AO, MFS, GBS, and Bickerstaff's brainstem encephalitis represent a continuous spectrum of conditions with a common pathogenesis. ${ }^{3}$ Anti-GQlb antibody positive MFS have been shown to affect corticospinal tract function reversibly in a previous study. ${ }^{4}$ Anti-GQlb antibodies have been shown to produce structural and functional changes at the neuromuscular junction in both ex vivo and in vitro studies. ${ }^{5}$ Although the neuromuscular junction is believed to be a site of action of these antibodies, this has only been deduced indirectly from electrophysiological studies. ${ }^{67}$ Single fibre electromyography (SFEMG) is regarded as the most sensitive method for assessing neuromuscular transmission defects. ${ }^{89}$ In this study, we report the use of stimulated SFEMG for serial evaluation of neuromuscular transmission in anti-GQlb antibody positive cases of acute neuromuscular conditions. The study's aim was to determine electrophysiological evidence to substantiate a defect at the neuromuscular transmission $^{10}$ and explain some of the motor effects of the MFS and its variants, in particular, those presenting as AO.

In this prospective study, we describe nine consecutive cases presenting with acute ophthalmoplegia over a one and a half year period, mostly in the absence of other clinical features. Serial SFEMG findings and other electrophysiological studies are discussed in relation to clinical and pathophysiological issues.

\section{CASE REPORTS}

Case one

A 49 year old man was admitted with a one day history of double vision and difficulty walking. Apart from some difficulty speaking, there was no dysphagia, vertigo bladder, or bowel symptoms. He had hypertension, which was well controlled with atenolol, and experienced symptoms of upper respiratory tract infection 10 days prior to admission.

On examination, he was alert and rational but had minimal dysarthria which resolved in the next three days. Eye movements were markedly reduced in all directions. He was generally hyporeflexic and had bilateral upper limb dysmetria. Motor power was not reduced apart from minimal proximal lower limb weakness. Facial muscle weakness was not evident. Gait was broad based and unsteady. A magnetic resonance imaging (MRI) brain scan was unremarkable. He improved rapidly over the next two weeks and was discharged with mildly reduced eye movements and mild dysmetria.

At the next review three months later, he was neurologically well and had full external ocular movements in all directions. His tendon reflexes had recovered and there was no detectable motor weakness. He was able to tandem walk steadily.

Anti-GQlb IgG titre was 28080 (normal <534) on admission but this reduced to 1706 during review at two months.

\section{Case two}

A 45 year old man with no significant past medical history was admitted with diplopia of two days in duration. He denied any change in voice, swallowing difficulty, weakness, or numbness of his limbs. There was no preceding fever,

Abbreviations: $A O$, acute ophthalmoparesis; ELISA, enzyme linked immunosorbent assay; EMG, electromyography; GBS, Guillain-Barré syndrome; MCD, mean consecutive differences; MFS, Miller Fisher syndrome; MRI, magnetic resonance imaging; NCS, nerve conduction studies; OD, optical density; PBS, phosphate buffered saline; RNS, repetitive nerve stimulation; SFEMG, single fibre electromyography 


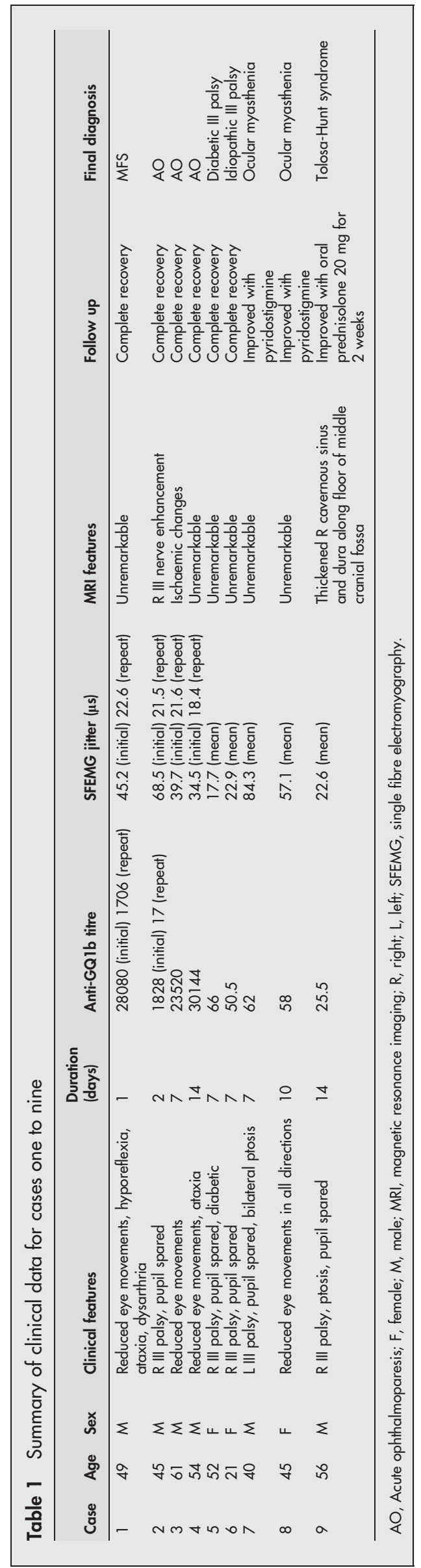

upper respiratory symptoms, gastrointestinal symptoms, visual aura, or headache. He did not drink or smoke.

Examination revealed that he was alert and orientated. There was no nuchal rigidity, conjunctival injection, or proptosis. Partial, non-fatigable ptosis in the right eye and impaired adduction, elevation, and downward eye movement were noted. There was no pupillary involvement. Examination of other cranial nerves and motor power was unremarkable. Deep tendon reflexes were normal. The physical signs were consistent with a pupil-sparing right III palsy. Further neurological examination did not reveal ataxia, sensory deficits to touch, pain, temperature, and abnormal position sense. He walked with a normal gait.

A brain MRI showed enhancement of the right oculomotor nerve in the interpeduncular cistern. The V, VII, and VIII complexes did not enhance and no ischaemic changes were seen on T2-weighted sequences. There were no brainstem MR abnormalities. Cerebrospinal fluid examination revealed absence of cells, elevated total protein of $12 \mathrm{~g} / \mathrm{l}$, and presence of globulin. Anti-GQIb IgG titre was significantly elevated at 1828 (normal <534).

Follow up at three months showed complete clinical recovery of the right III palsy. Again, a complete neurological examination did not reveal any deficits. The repeat anti-GQ1b antibody titre at this stage was only 17.

\section{Case three}

A 61 year old man complained of double vision for a week. He had a history of diabetes mellitus diagnosed six months ago and was on oral medication. There was no history of dysarthria, sensory symptoms, or motor weakness.

Clinical examination was unremarkable apart from reduced extraocular movements in all directions bilaterally. No ptosis, facial asymmetry, or weakness was obvious. The deep tendon reflexes were preserved. No clinical ataxia was elicited.

An MRI brain scan was unremarkable apart from incidental ischaemic changes in the left basal ganglia. Cerebrospinal fluid examination showed absent cells, elevated total protein of $0.5 \mathrm{~g} / \mathrm{l}$, and presence of globulin.

Acetylcholine receptor antibody titre was $0.12 \mathrm{nmo} / \mathrm{l}$ (normal $<0.25$ ). Anti-GQlb IgG titre was markedly elevated at 23520 (normal <534). Glycated haemoglobin concentration (HbAlc) was $6.9 \%$ (normal $<6.4 \%$ ). He was followed up and had complete recovery of eye movements at three months.

\section{Case four}

A 54 year old man had an upper respiratory tract infection, followed two weeks later by rapid onset of unsteady gait, diplopia, and giddiness. Clinical examination showed bilateral reduced eye movements in all directions, progressing onto near total ophthalmoplegia. Although there was no motor power weakness, mild and lower limb ataxia was present. There was no dysarthria, dysphagia, or hearing loss. Reflexes were preserved universally.

An MRI scan was unremarkable. Venereal Disease Research Laboratory (VDRL), thyroid function, and blood counts were normal. Cerebrospinal fluid examination did not show cells or raised proteins. Anti-GQlb IgG titre was markedly elevated at 30144 (normal <534). Upon follow up, his symptoms improved over two weeks and he had recovered his eye movements completely by six weeks.

Cases five to nine are summarised in detail in table 1.

Case five had elevated HbAlc at $11.4 \%$ (normal $<6.4 \%$ ). Case eight had elevated acetylcholine receptor antibody levels of $35 \mathrm{nmol} / \mathrm{l}$ (normal <0.25). None of these cases showed any features of bulbar palsy, motor weakness, hyporeflexia, or ataxia. 

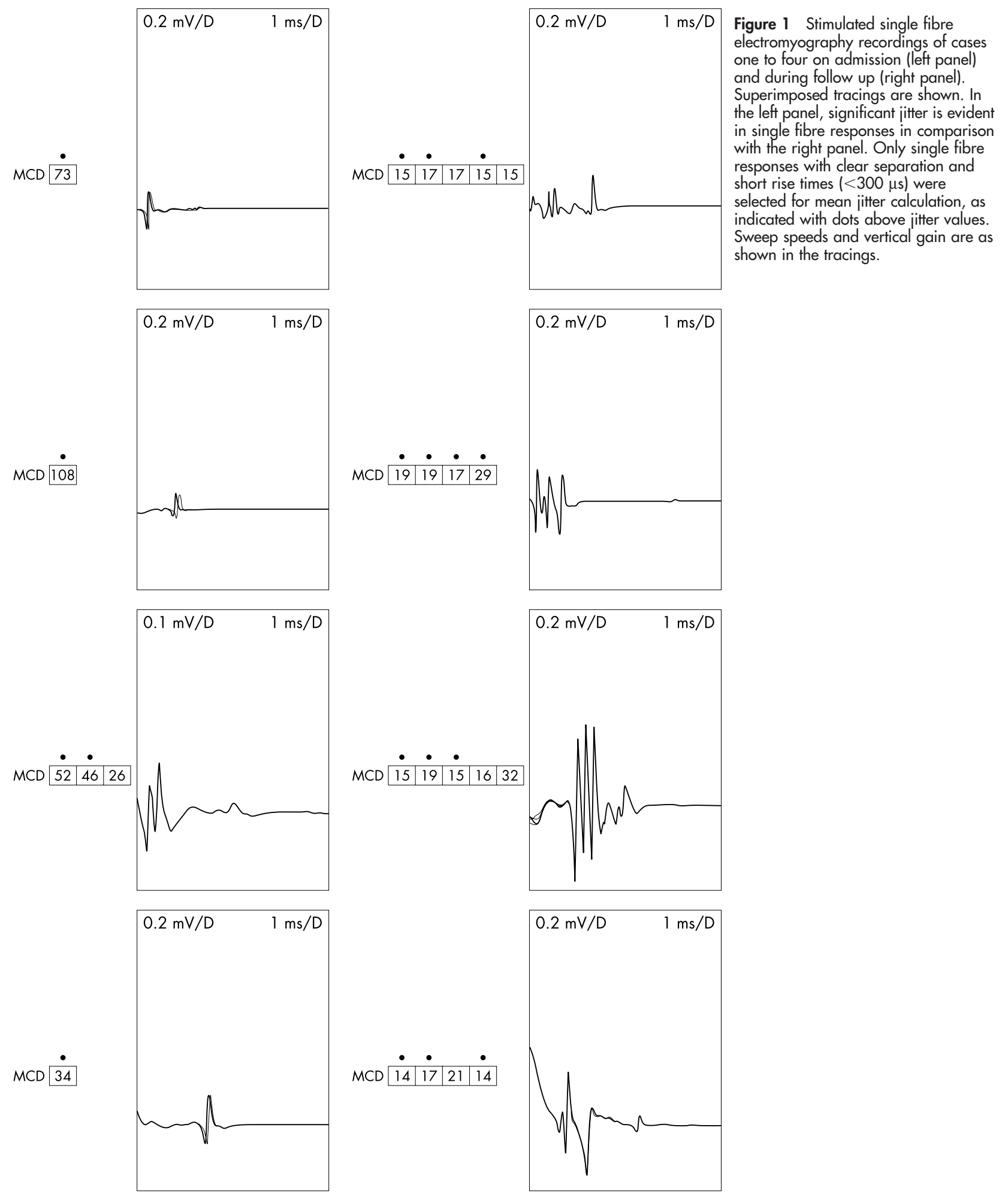

\section{METHODS}

Stimulated SFEMG was performed on the orbicularis oculi muscle of the affected eye at initial presentation (within one week of admission) and follow up at three months for antiGQ1b antibody positive cases. A Dantec 9013 K0872 needle (Dantec, Skovlunde, Denmark) $40 \mathrm{~mm}$ in length and $0.45 \mathrm{~mm}$ in diameter was inserted at the edge of the muscle for single fibre recordings. Axonal stimulation was achieved with a Teca disposable monopolar needle (Teca, Old Woking, Surrey, UK) placed $2.5 \mathrm{~cm}$ away from the recording needle

and a silver chloride disc as an anode at the malar prominence to produce visible twitches. Stimulation was achieved with square pulses of $0.04 \mathrm{~ms}$ duration at $10 \mathrm{~Hz}$ and intensity ranging from $8 \mathrm{~mA}$ to $15 \mathrm{~mA}$. Amplifier settings were fixed at 500 to $10 \mathrm{kHz}$. All studies were performed with a Dantec Keypoint Machine.

Stimulated single fibre responses were selected based on short rise times $(<300 \mu \mathrm{sec})$, clear separation from other discharges, and stable waveform morphology. For each study, individual mean consecutive differences (MCD) of 20 single 
fibre responses were averaged to obtain a final mean jitter value. Responses with MCDs less than $5 \mu$ sec are not included due to the likelihood of direct muscle fibre stimulation.

Before stimulated SFEMG, needle electromyography (EMG) was performed in the orbicularis oculi muscle at rest and during volitional activity with Medelec N 53153 concentric needles (Medelec, Old Woking, Surrey, UK).

Repetitive nerve stimulation (RNS) at $3 \mathrm{~Hz}$ with a square pulse $0.3 \mathrm{~ms}$ in duration of the orbicularis oculi, nasalis, trapezius, and abductor pollicis brevis muscles were studied. We utilised handheld Dantec 9013L0221 bipolar electrodes and 901350211 adhesive surface electrodes for stimulation and recording, respectively. Routine nerve conduction studies (NCS) of all four limbs, including VII nerve, " $\mathrm{F}^{\prime \prime}$ and " $\mathrm{H}$ " responses were performed after SFEMG studies.

Serum was assayed for antibodies to GQ1b ganglioside using enzyme linked immunosorbent assay (ELISA) methodology. Substrates were obtained from commercial sources (Sigma, St Louis, USA). $300 \mathrm{ng}$ of GQlb in $50 \mu \mathrm{l}$ of methanol was added to microtitre plate wells and evaporated to dryness to coat the wells. Any remaining binding sites were blocked with $100 \mu \mathrm{l}$ of $1 \%$ bovine serum albumin in PBS (phosphate buffered saline) for $4 \mathrm{~h}$ at room temperature. After washing with buffer, $100 \mu \mathrm{l}$ volumes of sera diluted 1:100 in PBS were added to the wells and incubated overnight at $4^{\circ} \mathrm{C}$. Samples were tested in duplicates with each run. The binding of anti-GQlb antibodies was measured by applying peroxidase conjugated goat anti-human IgG and developing with hydrogen peroxide and $0.1 \%$ O-phenylenediamene in citrate buffer. The reaction was stopped with $1 \mathrm{~N}$ sulphuric acid after $30 \mathrm{~min}$. Optical density (OD) readings were made at $492 \mathrm{~nm}$ using a Tecan Elisa reader. If the OD reading exceeded the upper limit of normal, the ELISA test was repeated at further dilutions to determine the dilutions at which OD readings were within the linear portion of the curve. The titre was then calculated by using the OD reading taken at the linear portion of the curve, extrapolating it to the expected OD value for serum dilution of $1: 100$ and multiplying by 1000 . Normal values for OD values and antibody titres were obtained from ELISA of sera from 56 normal control subjects. OD values and antibody titres greater than three standard deviations (SD) above the mean of the controls were considered abnormally elevated. OD values based normal controls yielded a mean of 0.234 and SD of 0.1 . The upper limit of normality (mean $+3 \mathrm{SD}$ ) was 0.534 . This corresponded to an antibody titre of 534.

\section{RESULTS}

Case one had an initial mean jitter of $45.2 \mu$ s and a jitter of $22.6 \mu$ s at three months (fig 1). NCS was unremarkable apart from absent " $\mathrm{H}$ " responses. Case two had an initial mean jitter of $68.5 \mu \mathrm{s}$ and a repeat jitter value of $21.5 \mu \mathrm{s}$. NCS was unremarkable apart from absent " $\mathrm{H}$ " responses. Case three had an initial mean jitter of $39.7 \mu$ s and a repeat jitter value of $21.6 \mu$ s at three months. Again, NCS was unremarkable. Case four's initial SFEMG study showed a mean jitter of $34.5 \mu \mathrm{s}$ and a repeat jitter value of $18.4 \mu$ s three months later. NCS showed absent " $\mathrm{H}$ " responses only.

Cases five to nine had mean jitters of 17.7, 22.9, 84.3, 57.1, and $22.6 \mu \mathrm{s}$, respectively. Upper limit of mean jitter was $23 \mu \mathrm{s}$ based on a control group of 20 normal subjects from our laboratory.

Additionally, none of the nine cases showed significant decremental responses on RNS or abnormal facial NCS or EMG.

\section{DISCUSSION}

All nine cases presented with rapid onset of acute ophthalmoplegia, mainly with absence of other clinical features. Complete recovery was seen over a three month period and no recurrence was documented.

Cases one to four had significantly elevated anti-GQlb antibody titres at initial presentation. Initial SFEMG studies showed abnormal mean jitters, which improved markedly over the three month period, in tandem with clinical recovery. Neuroimaging was unremarkable apart from case two. The levels of anti-GQlb antibody appeared to have some correlation with the degree of ocular involvement. Case two, with a modest elevation of anti-GQlb antibody titre, only showed signs of unilateral III palsy, in contrast to cases one, three, and four, which had reduced external ocular movements in all directions. Case one largely fulfilled criteria for MFS, considered a major subset of the "anti-GQ1b syndrome" spectrum.

In $\mathrm{AO}$, both unilateral and bilateral ocular palsy has been observed. ${ }^{11}{ }^{12}$ The III, IV, and VI cranial nerves have been shown to possess significantly higher percentages of GQlb than other cranial nerves and this lends support to the role of anti-GQlb in the pathogenesis of ophthalmoplegia. ${ }^{13}$ In case two, the enhancement of the right III nerve, as with previous reports of $\mathrm{IV}^{14} \mathrm{~V},{ }^{15}$ and $\mathrm{VI},{ }^{16}$ is likely to have a similar immunological pathogenesis.

Case five was clinically diagnosed as having diabetic related III palsy. Case six had an idiopathic partial III palsy with normal SFEMG and anti-GQIb antibody titres. Case seven was diagnosed and managed as ocular myasthenia gravis with marked clinical improvement, as was case eight. Case nine was managed as a Tolosa-Hunt syndrome variant, supported by MRI findings. ${ }^{17-19}$ Cases five to nine serve as incidental negative controls for cases one to four both clinically and electrophysiologically. They illustrate the high sensitivity of SFEMG for neuromuscular transmission defects but lack of specificity as seen in cases seven and eight. However, SFEMG abnormalities appear to mirror anti-GQlb antibody titres, as with case six. Despite the possibility of cranial neuropathy in cases five and nine, normal SFEMG results support its specificity for diagnosing neuromuscular junction abnormalities rather than abnormalities attributed to demyelinating or axonal neuropathy.

The orbicularis oculi was selected for SFEMG study for reasons of ease of stimulation, availability of normal values, and proximity to extraocular muscles. The latter reasoning is based on RNS studies in myasthenia gravis, another condition with impaired neuromuscular transmission, where a proximal muscle close to the area of maximal weakness is more likely to show electrophysiological abnormalities. ${ }^{20} 21$ The normal facial nerve conduction and lack of active denervation changes in the orbicularis oculi muscles do not favour the presence of significant VII neuropathy in all cases.

In this paper, cases one to four illustrate dynamic improvement in mean jitter in tandem with clinical recovery. The evidence for neuromuscular transmission impairment in MFS is well documented in vitro. Anti-GQlb antibody related effects on the synaptic region, ${ }^{22}$ with complement mediation ${ }^{23}$ and structural injury at the nerve terminal, ${ }^{24}$ are recent findings. Although rapid reversibility of presynaptic blocking actions of these antibodies have been demonstrated, ${ }^{25}$ the exact electrophysiological abnormalities remains uncertain, and mild transient blocking action of antibodies to the presynaptic or postsynaptic regions cannot be completely excluded. Most recently, it has also been shown that in the absence of complement, monoclonal antibody to anti-GQlb IgM depressed evoked quantal release of acetylcholine dosedependently at the presynaptic region in mice hemidiaphragms. $^{26}$ 
The action of anti-GQlb antibody on neuromuscular transmission in each case can be deduced from in vitro studies. Patch clamp techniques have demonstrated reversible pre and postsynaptic neuromuscular blocking effects of circulating IgG antibodies in MFS sera. ${ }^{27}$ More specifically, both failure of acetylcholine release $\mathrm{e}^{25} 28$ and massive quantal acetylcholine release ${ }^{29}$ causing a "depolarising block" effect has been demonstrated. As all these processes have been shown to be reversible, they likely contributed to reversible neuromuscular transmission failure detected with the sensitive technique of SFEMG in our study.

It was argued that despite in vitro documentation of neuromuscular blockade in MFS, neurophysiological evidence to substantiate this, unlike in myasthenia gravis, is lacking. ${ }^{10}$ This is the first paper demonstrating with SFEMG the dynamic improvement of neuromuscular transmission in anti-GQ1b antibody positive cases of $\mathrm{AO}$ in tandem with clinical recovery. It also highlights the value of SFEMG in differentiating weakness due to neuromuscular transmission defect as opposed to neuropathy in certain clinical situations. Future studies correlating clinical features, SFEMG findings and serial antiganglioside antibody titre levels would certainly be of interest.

\section{ACKNOWLEDGEMENTS}

The authors thank Dr WC Yee and Ms Chen Yan for help with the anti-GQlb antibody assays.

\section{Authors' affiliations}

Y L Lo, A Pan, P Ratnagopal, Department of Neurology, National

Neuroscience Institute, Singapore General Hospital, Singapore L L Chan, Department of Diagnostic Radiology, Singapore General Hospital, Singapore

Competing interests: none declared

\section{REFERENCES}

1 Sauron B, Bouche P, Canthala HP, et al. Miller Fisher syndrome: clinical and electrophysiological evidence of peripheral origin in 10 cases. Neurology 1984;43:953-6.

2 Yuki N, Odaka M, Hirata K. Acute ophthalmoparesis (without ataxia) associated with anti-GQ1b lgG antibody: clinical features. Ophthalmology 2001; 108:196-200

3 Odaka M, Yuki N, Hirata K. Anti-GQ1b antibody syndrome: clinical and immunological range. J Neurol Neurosurg Psychiatry $2001 ; 70: 50-5$.

4 Lo YL, Ratnagopal P. Transcranial magnetic stimulation studies in the Miller Fisher syndrome: evidence of corticospinal tract abnormality. I Neurol Neurosurg Psychiatry 2001;71:210-4.

5 Slater C. Double agents and breakdown of integrity at the neuromuscular junction in Miller Fisher syndrome. Brain 2001;124:847-8.

6 Uncini A, Lugaresi A. Fisher syndrome with tetraparesis and antibody to GQ1b: evidence for motor nerve terminal block. Muscle Nerve 1999;22:640-4.

7 Pullicino P, Beck N. Incremental response to repetitive stimulation in GuillainBarre syndrome. J Neurol Neurosurg Psychiatry 1992;55:233-4.
8 Padua L, Stalgerg $E$, LoMonaco $M$, et al. SEMG in ocular myasthenia diagnosis. Clin Neurophysiol 2000;111:1203-7.

9 Sonoo M, Uesugi $H$, Mochizuki A, et al. Single fibre EMG and repetitive nerve stimulation of the same extensor digitorium communis muscle in myasthenia gravis. Clin Neurophysiol 2001;112:300-3.

10 Winer JB. Bickerstaff's encephalitis and the Miller Fisher syndrome. J Neurol Neurosurg Psychiatry 2001;71:433-5.

11 Kuroki S, Saida T, Nukina M, et al. Three patients with ophthalmoplegia associated with Campylobacter jejuni. Pediatr Neurol $2001 ; 25: 71-4$.

12 Caccavale A, Mignemi L. Acute onset of a bilateral areflexical mydriasis in Miller Fisher syndrome: a rare neuro-ophthalmologic disease. J Neuroophthalmol 2000;20:61-2.

13 Chiba A, Kusunoki S, Obata H, et al. Serum anti-GQ1b lgG antibody is associated with ophthalmoplegia in Miller Fisher syndrome and Guillain-Barre syndrome: clinical and immunohistochemical studies. Neurology 1993:43:1911-17.

14 Tanaka H, Yuki N, Hirata K. Trochlear nerve enhancement on threedimensional magnetic resonance imaging in Fisher syndrome. Am J Ophthalmol 1998;126:322-4.

15 Farah S, Khuraibet AJ, Montaser MA, et al. Miller Fisher syndrome: unique involvement of ophthalmic division of trigeminal nerve. Clin Neurol Neurosurg 1995;97:328-31.

16 Shibata A, Hosoya T, Kato T, et al. Abducens nerve enhancement in acute ophthalmoparesis. Radiat Med 1998;16:375-7.

17 Pascual J, Cerezal L, Canga A, et al. Tolosa-Hunt syndrome: focus on MRI diagnosis. Cephalalgia 1999;19:36-8.

18 Goto Y, Hosokawa S, Goto I, et al. Abnormality in the cavernous sinus in three patients with Tolosa-Hunt syndrome: MRI and CT findings. J Neurol Neurosurg Psychiatry 1990;53:231-4.

19 Yousem DM, Atlas SW, Grossman RI, et al. MR imaging of Tolosa-Hunt syndrome. Am J Roentgenol 1990;154:167-70.

20 Krarup C. Electrical and mechanical responses on the platysma and the adductor pollicis muscle in normal subjects. I Neurol Neurosurg Psychiatry 1977;40:234-40.

21 Oey PL, Wieneke GH, Hoogenraad TU, et al. Ocular myasthenia gravis: the diagnostic yield of repetitive nerve stimulation and stimulated single fibre EMG of the orbicularis muscle and infrared reflection oculography. Muscle Nerve 1993;16:142-9.

22 Wessig C, Buchwald B, Toyka KV, et al. Miller Fisher syndrome: immunofluorescence and immunoelectron microscopic localisation of $\lg G$ at the mouse neuromuscular junction. Acta Neuropathol (Berl) $2001 ; 101: 239-44$.

23 Bullens RW, O'Hanlon GM, Goodyear CS, et al. Anti-GQ1b antibodies and evoked acetylcholine release at mouse endplates. Muscle Nerve 2000;23:1035-43.

24 O'Hanlon GM, Plomp JJ, Charabarti M, et al. Anti-GQ1b ganglioside antibodies mediate complement-dependent destruction of the motor nerve terminal. Brain 2001;124:893-906.

25 Buchwald B, Weishaupt A, Toyka KV, et al. Immunoglobulin G from a patient with Miller-Fisher syndrome rapidly and reversibly depresses evoked quantal release at the neuromuscular junction of mice. Neurosci Lett 1995;201:163-6.

26 Buchwald B, Ahangari R, Toyka KV. Differential blocking effects of the monoclonal anti-GQ1b IgM antibody and alpha-latrotoxin in the absence of complement at the mouse neuromuscular junction. Neurosci Lett 2002;334:25-8.

27 Buchwald B, Bufler J, Carpo $M$, et al. Combined pre and postsynaptic action of IgG antibodies in Miller Fisher syndrome. Neurology 2001;56:67-74.

28 Roberts M, Willison H, Vincent A, et al. Serum factor in Miller-Fisher variant of Guillain-Barre syndrome and neurotransmitter release. Lancet 1994;343:454-5.

29 Jacobs BC, Bullens RW, O'Hanlon GM, et al. Detection and prevalence of alpha-latrotoxin-like effects of serum from patients with Guillain-Barre syndrome. Muscle Nerve 2002;25:549-58. 